\title{
SHG/2PF microscopy of single and multi-layer graphene
}

\author{
Alexander V. Klekachev a,b, Inge Asselberghs ${ }^{\mathrm{a}, \mathrm{c}}$, Cedric Huyghebaert ${ }^{\mathrm{a}}$, Maarten Vanbel ${ }^{\mathrm{c}}$, Monique A. \\ Van der Veen ${ }^{\mathrm{c}}$, André L. Stesmans ${ }^{\mathrm{b}}$, Marc M. Heyns ${ }^{\mathrm{a}, \mathrm{d}}$, Stefan De Gendt ${ }^{\mathrm{a}, \mathrm{c}}$ and Thierry Verbiest ${ }^{\mathrm{c}}$ \\ aimec, Kapeldreef 75, B-3001 Leuven, Belgium; \\ ${ }^{\mathrm{b}}$ Department of Physics and Astronomy, Katholieke Universiteit Leuven, Celestijnenlaan 200D, B- \\ 3001 Leuven, Belgium \\ ${ }^{c}$ Department of Chemistry, Katholieke Universiteit Leuven, Celestijnenlaan 200F, B-3001 Leuven, \\ Belgium \\ ${ }^{\mathrm{d}}$ Department of Metallurgy and Materials Engineering, Katholieke Universiteit Leuven, Kasteelpark \\ Arenberg 44, B-3001 Leuven, Belgium
}

\section{Abstract}

Since the discovery of graphene in 2004 by Novoselov and Geim, a lot of research emphasis has been directed towards its characterization. Most of the important scientific breakthroughs have been obtained on exfoliated graphene (produced via the well known 'scotch tape' method), nowadays, different synthetic routes have been developed to obtain large-scale graphene. Among several optical techniques, Raman spectroscopy is the one most often employed to characterize the defects, number of graphene layers and other properties of the graphitic films regardless of their fabrication method. In this work, we will report on the microscopic imaging of the two-photon fluorescence (2PF) properties and the second-harmonic generation (SHG) in both single layer and few layer graphene.

\section{Introduction}

From the family of all carbon materials, built only from sp2 hybridized C-atoms, graphene is discovered the latest and it is the 2 dimensional allotrope, being only one single atom thick[1]. The discovery was achieved by Novoselov and Geim in 2004, by basically peeling of a single layer from natural graphite using adhesive tape[2]. This method is referred to as the scotch tape method, and is still the most employed method, due to its high accessibility. It also results in achieving of highest quality graphene samples available so far. The increased research boost after 2004, revealing many intriguing and unique properties, such as strong field effect, high carrier mobilities and low sheet resistance[3-4], only increased the need for synthetically grown graphene. Different methods are currently available for growing graphene layers in artificial conditions obtaining large uniform areas. However, difficulties are still encountered due to the presence of grain boundaries and defects in the graphene lattice in synthesized obtained graphene. These anomalies alter some of the unique properties observed in exfoliated material. Another degree of variation of graphene properties is number of layers and their stacking. The latter can result in different electronic structure as has been recently evidenced in three-layer graphene by Raman scattering spectroscopy[5] and electrical characterization in transistor configuration[6]. Nowadays, a broad variety of possible applications are screened, as well as large emphasis is put into fully understand the behavior. Yet, second harmonic generation (SHG) and two-photon luminescence (2PF) imaging are capable of providing structural information with spatial resolution. Examples are the organization of nanoparticles[7-8] and host/guest systems[9-10]. Even point group symmetry determination is possible based on nonlinear optical microscopy alone[11-13]. One of the fields, which is yet limited explored, is the field of nonlinear optical imaging. In this work, we are combining for the first time the results obtained from both second-harmonic generation and two-photon luminescence (2PF) imaging for graphene. We report on the results obtained for single layer and bilayer graphene, as well as the difference obtained for pristine and synthetic material. 


\section{Experimental techniques}

\subsection{Sample preparation}

Two types of graphene samples are prepared and compared in this work; the exfoliated graphene, produced by micromechanical cleavage of natural graphite and graphene produced by chemical vapor deposition (CVD). In both cases, graphene was positioned onto standard $170 \mu \mathrm{m}$ thick microscope coverslips required to fulfill the experimental setup conditions.

\subsubsection{Exfoliated graphene samples}

The exfoliated graphene samples are obtained after applying the classical scotch tape method[2], in which a piece of the graphite is thinned with a sharp razor blade. A small part is pealed of by the tape, and is uniformly redistributed over the tape by softly double folding and releasing the tape. (See Scheme 1) After obtaining a dense graphite film over the tape, the tape is pushed onto the glass substrate and released slowly. By carefully inspecting the sample by means of an optical microscope, the graphene pieces of different thickness are detected. A typical example of an exfoliated graphene sample on a glass slide is shown in Scheme 1(d) in which the assignment of the number of layers is done based on the optical contrast difference. This was later on confirmed by Raman spectroscopy. (See section 2.2).

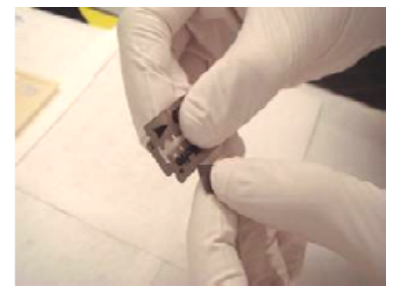

(a)

Thinning the graphite by razor blade

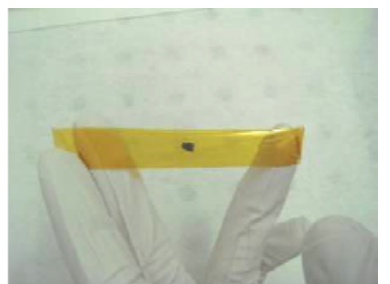

(b)

Transferring a small graphite piece to tape

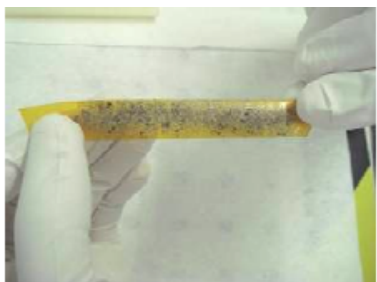

(c)

Uniformly redistributing the graphite over the tape

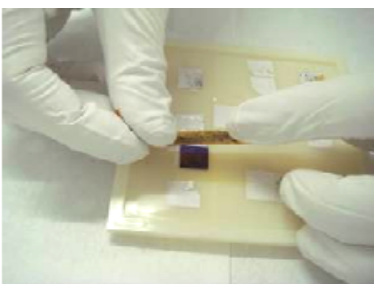

(d)

Gently transfer the graphite/graphene to substrate

Scheme 1: Schematic representation of preparation method of the exfoliated samples.

\subsubsection{Transferred CVD graphene samples}

The synthetic graphene samples were grown by chemical vapor deposition (CVD) on copper, and were provided by Prof. Cho (KAIST, Korea) using their standard procedure as reported in [14-15]. For transferring the graphene from the $\mathrm{Cu}$ surface to the glass substrate the following method was used; the $\mathrm{Si} / \mathrm{SiO} 2 / \mathrm{Cu} / \mathrm{Graphene}$ stack is covered with a thin Poly(methyl methacrylate) (PMMA) film. The $\mathrm{Cu} /$ Graphene/PMMA stack is then extracted from the source wafer with a help of a piece of adhesive tape attached to a small corner of the substrate. The as such obtained floating layer of $\mathrm{Cu} / \mathrm{Graphene} / \mathrm{PMMA}$ is transferred to a Cu-etching solution of $0.1 \mathrm{M} \mathrm{FeCl}$ during 15 min. After etching the PMMA/graphene layer is transferred to deionized water for several times in order to remove the residual ions (Scheme 2). The resulting Graphene/PMMA film is transferred onto the glass coverslip and, finally, the PMMA film is removed by putting the sample in acetone $\left(50^{\circ} \mathrm{C}\right)$ during $12 \mathrm{~h}$ followed by rinsing in isopropyl alcohol (IPA) and N2 drying step.

\section{$\mathrm{Si} / \mathrm{SiO}_{2} / \mathrm{Cu} /$ Graphene source wafer}

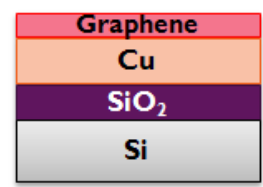

PMMA deposition

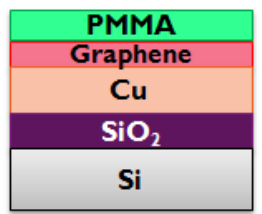

Transfer of Cu/Graphene/PMMA film to $\mathrm{Cu}$ etchant

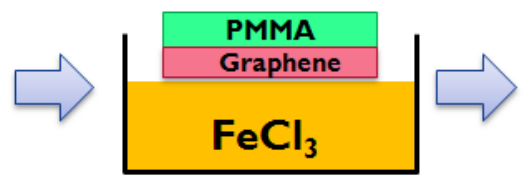

Transfer to glass substrate and PMMA strip

Glass coverslip

Scheme 2: Schematic representation of graphene transfer techniques employed in this work. 


\subsection{Raman spectroscopy}

Raman scattering spectroscopy is a powerful technique in terms of studying graphene properties[16]. Graphene has a very specific unique Raman fingerprint. It shows a narrow band around $1585 \mathrm{~cm}^{-1}$; which is assigned to the stretching modes of the $\mathrm{sp}^{2} \mathrm{C}$ bonds, and is referred to as $\mathrm{G}$ band. A second feature is the breathing mode of the $\mathrm{sp}^{2} \mathrm{C}$ bonds and results in a very sharp D and/or 2D band, around 1250 and $2650 \mathrm{~cm}^{-1}$, respectively. The 2D band is the second-order of the $\mathrm{D}$ band and has a specific shape depending on the number of interacting graphene layers. In case of single layer graphene the 2D band is very sharp, while in case of a bilayer system, the band is broader and can only by fitted to four Lorentzian curves. In a perfect graphene lattice, the $\mathrm{D}$ band is absent, while a large intensity is representative for highly defective graphene. The intensity ratio of $D$ to $G\left(I_{D} / I_{G}\right)$ is often used as a measure of crystalline quality of graphene, while $2 \mathrm{D}$ to $\mathrm{G}$ ratio $\left(\mathrm{I}_{2 \mathrm{D}} / \mathrm{I}_{\mathrm{G}}\right)$ is typically above unity for undoped graphene[17-18]. Raman spectra of graphene films deposited onto the glass substrate were characterized by means of conventional confocal micro-Raman spectrometer (HORIBA Jobin Yvon LabRam 800). The excitation light of $532 \mathrm{~nm}$ DPSS laser was focused into a spot with a diameter of $0.7 \mu \mathrm{m}$ and safe enough power density of $30 \mathrm{~kW} / \mathrm{cm}^{2}$ [19]. In Fig 2.1 a typical graphene spectrum is represented for both single and bilayer graphene. One notice immediate the absence of the D peak, proving the good crystalline quality of the graphene sample.
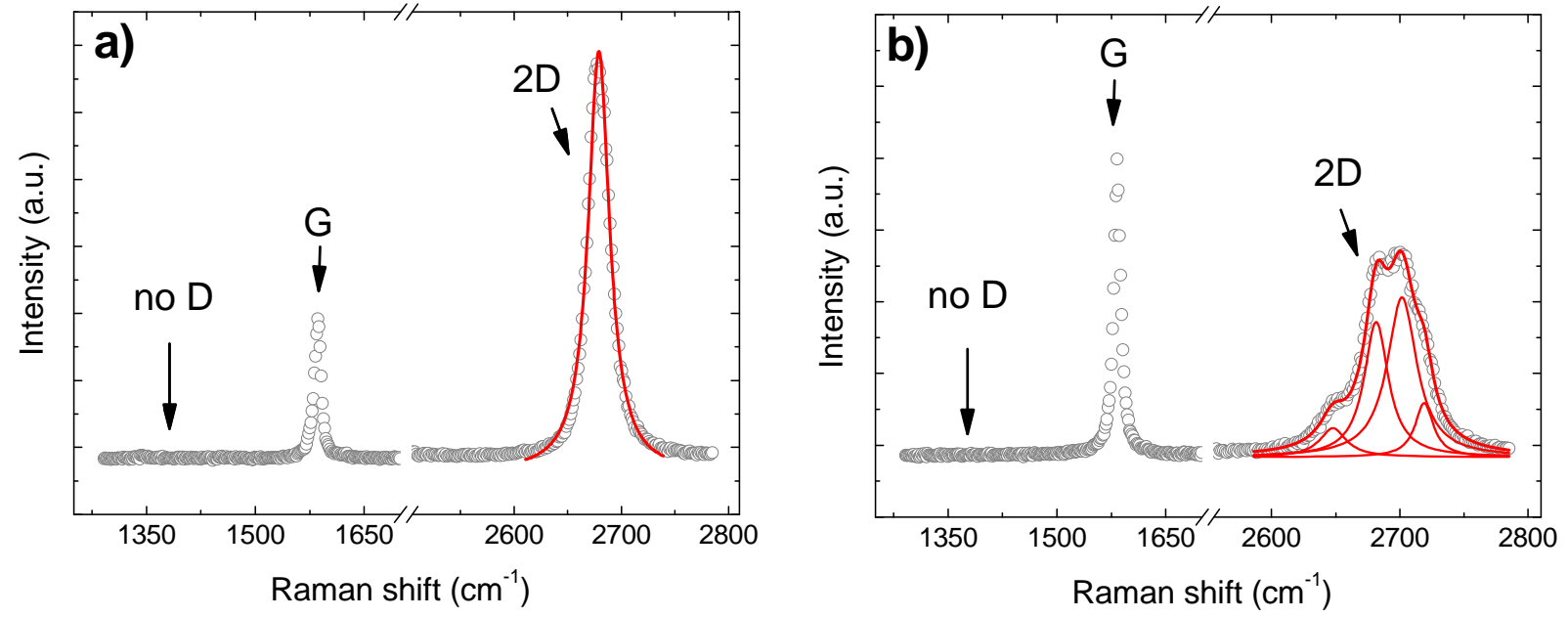

Fig. 2.1: Raman spectrum of a typical graphene sample for (a) single layer and (b) bilayer graphene measured on exfoliated graphene samples.

For the transferred CVD graphene samples, both Raman spectroscopy and AFM images are performed in order to confirm the quality of the graphene layer after transfer. In each sample a sharp and highly intense $2 \mathrm{D}$ peak was observed $\left(\mathrm{FWHM}=38.53 \pm 1.54 \mathrm{~cm}^{-1}\right)$ confirms the single layer nature of the sample[16]. The presence of weak D-peak suggests low defect density and considerable crystalline quality of the graphene lattice over the whole flake area. Both ratios averaged over several samples are $\mathrm{I}_{2 \mathrm{D}} / \mathrm{I}_{\mathrm{G}}=1.77 \pm 0.17$ and $\mathrm{I}_{\mathrm{D}} / \mathrm{I}_{\mathrm{G}}=0.18 \pm 0.05$. Additionally, we analyze the surface of transferred CVD graphene films employed in this work by atomic force microscopy (AFM) (Figure XX). The RMS surface roughness value was found around $0.51 \mathrm{~nm}$. Considering all these points, the transferred CVD graphene used in this work is of sufficient quality for usage in non-linear optical experiments. 


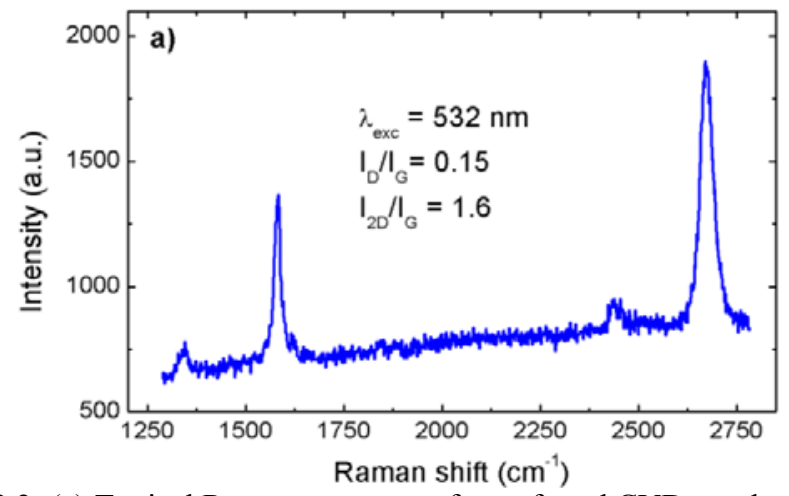

b)

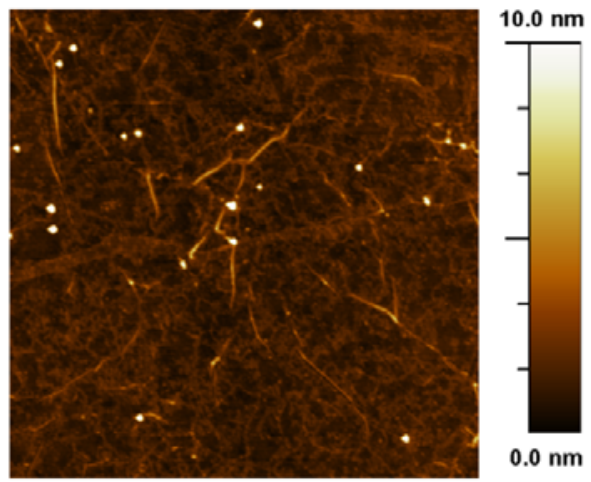

Fig. 2.2: (a) Typical Raman spectrum of transferred CVD graphene; (b) AFM image of the same sample; the image size is $3 \times 3 \mu \mathrm{m}^{2}$.

\subsection{Second-Harmonic Generation (SHG) and Two-Photon Fluorescence (TPF) Imaging}

The SHG and TPF images are obtained by using a home built set-up in which a high power femtosecond-pulsed laser is coupled to an olympus IX71 microscope. The laser beam of a Ti:sapphire laser (Spectra Physics, Tsunami), emitting at $\lambda_{0}=800 \mathrm{~nm}$ is focused by a long distance focusing lens $(\mathrm{f}=7.5 \mathrm{~cm})$, approximating the beam properties of a collimated beam, under normal incidence. The transmitted light is collected by a high power objective and focused onto the built in microscope EM-CCD camera (electron multiplying charge coupled device; Hamamatsu). To discriminate between the SHG and TPF signals, the appropriate band-pass filters are applied (400 $\mathrm{nm}$ and 420-650 nm, respectively). In the schematic representation of the set-up (Fig 2.2), the Z-axis corresponds to the normal incidence direction. In the recorded images, the horizontal and vertical direction corresponds to the $\mathrm{X}$ and $\mathrm{Y}$ - axis, respectively. The recorded polarization patterns are taken over $360^{\circ}$ in steps of $5^{\circ}$, by rotating a halve-wave plate. The complete description of the experimental set-up can be found in [20]. The experimental conditions are similar for all samples.
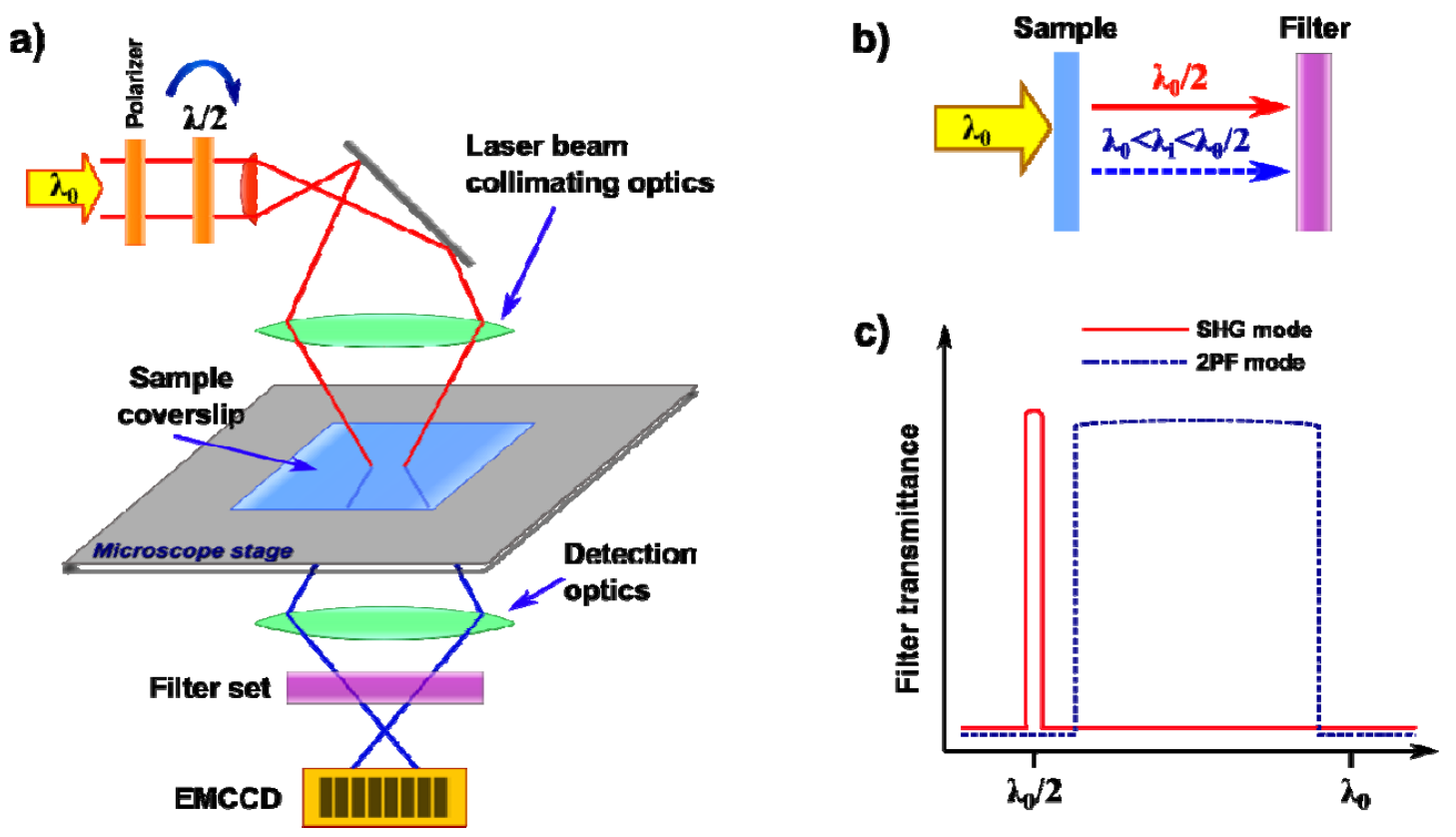

Fig 2.2: Schematic representation of the experimental set-up: (a) simplified setup structure; (b) a sketch showing difference between two types of experiments (SHG or 2PF modes) depending on the transmittance curve (c) of installed filters set. 


\section{Results and Discussion}

Initially, we start to record data from the exfoliated graphene samples. From the optical image (See fig 3.1(a)), we select a region of interest, which clearly shows thinner and thicker parts. The thinner regions are assigned to single layer graphene and the more dense part to bilayer graphene. This was confirmed by Raman spectroscopy (Fig. 3.1(b)), where an intensity map of the ratio of $2 \mathrm{D}$ and $\mathrm{G}$ is plotted. When illuminating the sample with $800 \mathrm{~nm}$ laser light, and collecting the emitted light in transmission in the optical window ranging from $420-650 \mathrm{~nm}$, a clearly distinct intensity response is observed, where the bilayer part is emitting at a higher intensity as compared to the single layer part.

a)

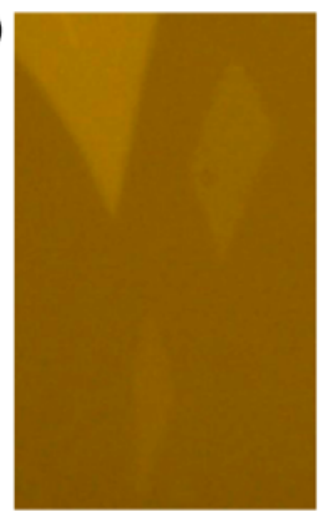

b)

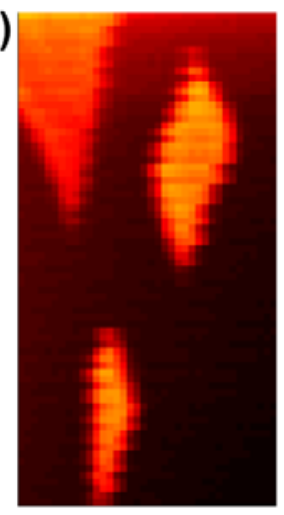

c)

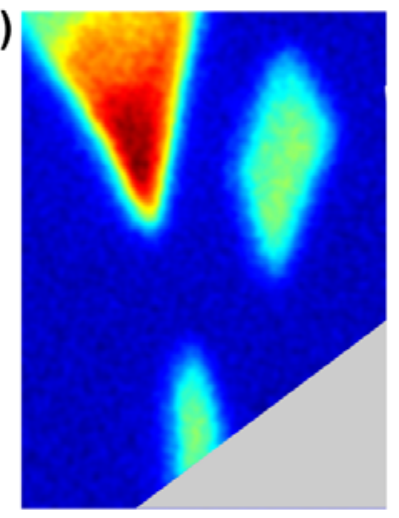

d)

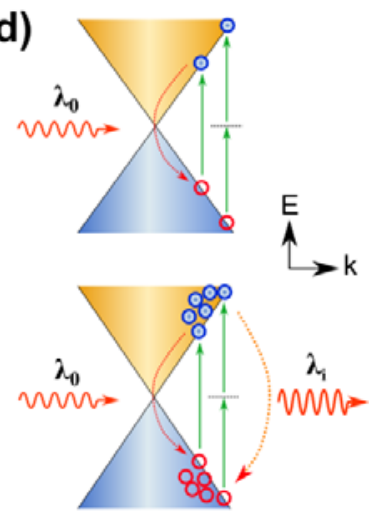

Fig. 3.1: (a) Optical image of single layer and bilayer graphene flakes; (b) Raman image of I2D/IG ratio; (c) Photoluminescence image; (d) Schematic of single and two-photon absorption followed by e-h pair generation and recombination in graphene for the case of continuous mode excitation (top) and pulsed pumping regime resulting in high-density electron-hole plasma formation (bottom).

Due to its semimetallic nature, graphene does not exhibit any photoluminescence response under continuous mode excitation: photoexcited electron-hole pair will immediately recombine nonradiatively (Figure 3.1(d), top). In order to make graphene flakes fluorescent, an energy gap has to be introduced like it has been demonstrated for $\mathrm{O}_{2}$-plasma treated graphene [21] and chemically derived graphene oxide sheets and solutions [22-23]. However, a periodic ultra short light pulse illumination/excitation pulse will create a non-equilibrium electron-hole plasma in graphene. This effect has been recently employed in graphene-based saturable light absorbers for mode-locking lasers [24-25]. A high-density electron hole plasma in graphene will also make radiative electron-hole process possible which is responsible for the observed photoluminescence as seen schematically in figure 3.1(d, bottom).

When assigning a certain pathway on the photoluminescence image (Fig 3.2a), and plotting the intensity of the response as a function of distance on this path (Fig 3.2b), a stepwise profile is observed. The steps observed, are consistent with the assignment of the single and bilayer graphene. This is consistent with data reported in the literature by Liu et al.[26] and Stohr at al. [27]; although reflection geometry is used in their experiments. In both reports, they demonstrate the simple additivity of the emission signals dependent on the number of layers. This allows us to quickly map a graphene sample in terms of the presence of the number of layers.

When applying the same procedure to a CVD transferred graphene sample (Fig 3.2(c)), a uniform illumination image is observed on the smooth single layer sample part, while focusing on a mechanical folded graphene edge region, a more intense signal is observed (Fig. 3.2(d)). Note that a correction for differences in input intensity needs to be applied due to non-uniform samples illumination. Although, further experiments should be performed to fully understand the spectroscopic response of both types of graphene sources. 

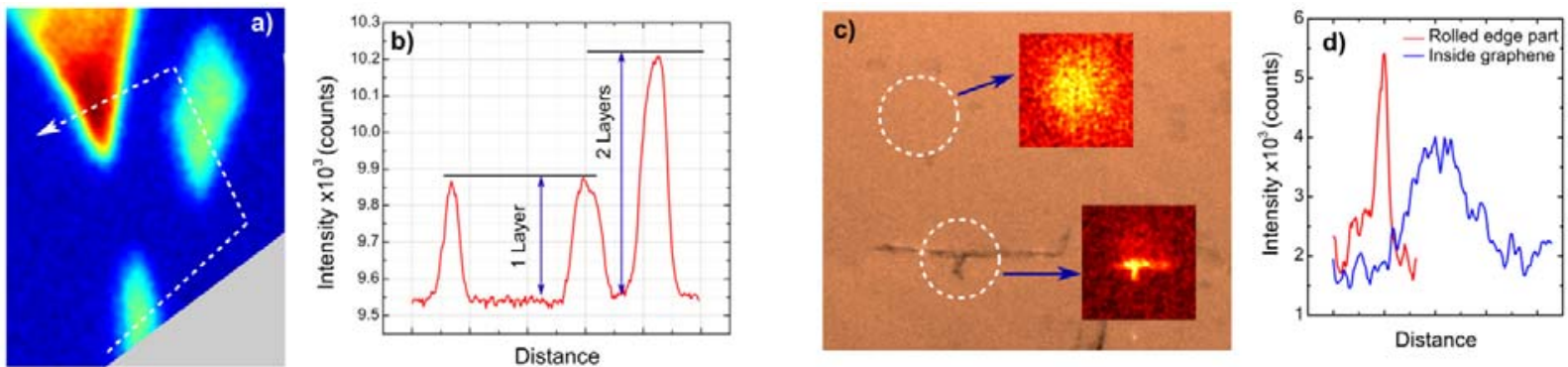

Fig. 3.2: (a) Photoluminescence image and its profile scan (b) shown by white dashed line; (c) Optical image of transferred CVD graphene film where insets show 2PF scans in the graphene area and at the rolled edge of graphene film. (d) Intensity profiles for $2 \mathrm{PF}$ signal inside graphene area and at the rolled edge part.

In a second experiment, we change the filter settings of the imaging microscope to the SHG mode. Note that, opposed to previous literature reports [28], we collect any SHG effect in the perpendicular input direction. Fig 3.3 shows the plot of the SHG response as a function of input polarization of the fundamental light. For the single layer graphene no clear polarization dependence is observed, while for the bilayer graphene sample, a 3-fould symmetry pattern is observed. Opposed to what is observed by Van Driel et al. in [28], a different polarization pattern is observed. However, one has to be careful to do a direct comparison since the data are collected in different experimental geometry. However, for the bilayer graphene, the same symmetry pattern is observed. In case of single layer graphene, a different polarization pattern is observed. Additional to a difference in measurement conditions, also different substrates are used, which have an influence on the SHG response.

Opposite to the observation of photoluminescence in the 420-620 nm region, no SHG signal is observed for the CVD transferred samples in the same experimental conditions as the exfoliated samples. It can be assumed/argued that this absence of SHG signal originates in $\mathrm{C}_{3}$-symmetry breaking of the carbon lattice due to the presence of defects and grain boundaries in the crystalline lattice of CVD graphene. Therefore, SHG intensity imaging can be employed as a fast defect mapping technique providing significantly better throughput than Raman scattering spectroscopy imaging.

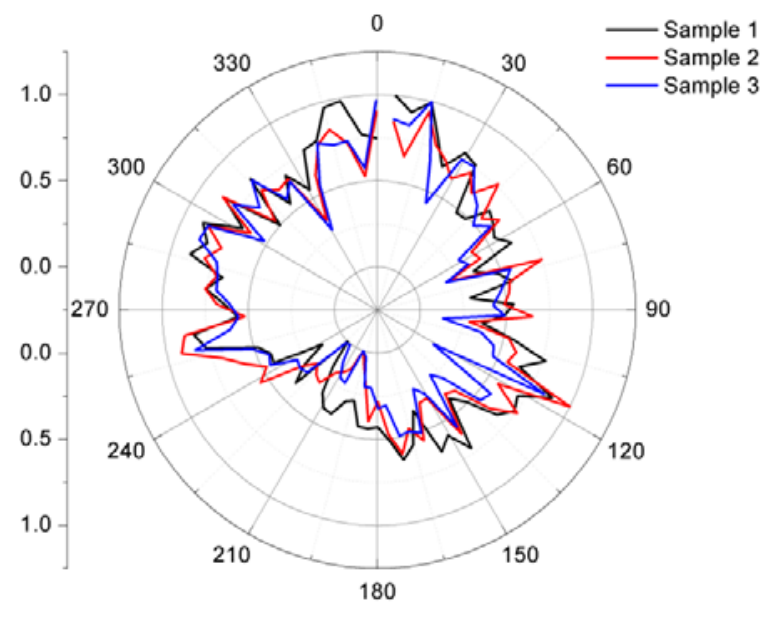

(a)

SHG intensity as a function of input polarization: Single layer graphene

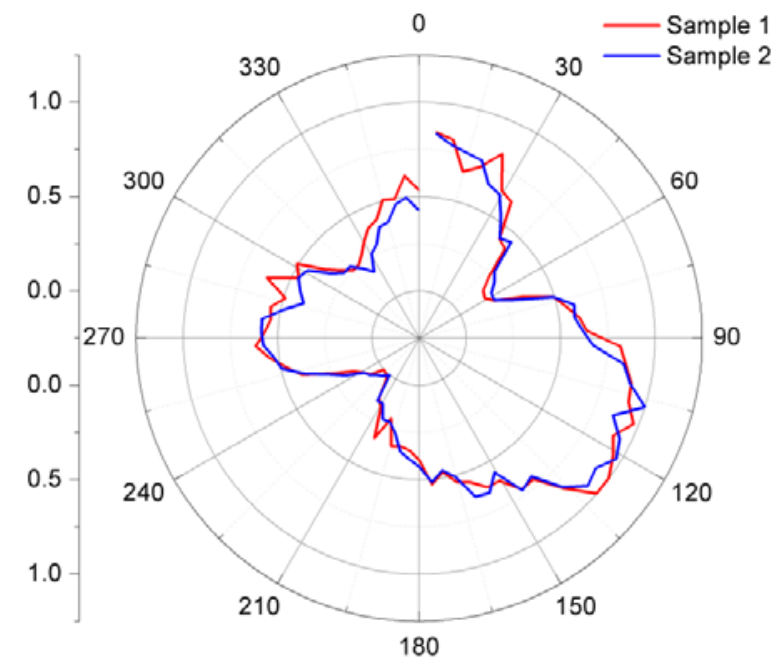

(b)

SHG intensity as a function of input polarization : bi-layer graphene 


\section{Conclusions}

We have performed an analysis of pristine graphene samples and compared the outcome with the response of CVD transferred graphene. In both type of samples, similar emission intensity is observed from single to bilayer/multilayers regions. We benchmark transferred CVD graphene towards exfoliated graphene by 2PF microscopy for the first time. On the other hand, for the second-harmonic signals, no effect is observed for CVD transferred graphene, while a different polarization pattern is observed for pristine samples. However, this work is not yet totally conclusive and more experiments will be required to fully understand the ongoing processes.

\section{Acknowledgements}

We would like to acknowledge Prof B.J. Cho from KAIST, Korea, for providing us with CVD grown graphene. MvdV thanks the Fund for Scientific Research-Flanders (FWO-V) for a post-doctoral fellowship.

\section{References}

[1] K. S. Novoselov, D. Jiang, F. Schedin et al., "Two-dimensional atomic crystals," Proceedings of the National Academy of Sciences of the United States of America, 102(30), 10451-10453 (2005).

[2] K. S. Novoselov, A. K. Geim, S. V. Morozov et al., "Electric field effect in atomically thin carbon films," Science, 306(5696), 666-669 (2004).

[3] A. H. Castro Neto, F. Guinea, N. M. R. Peres et al., "The electronic properties of graphene,” Reviews of Modern Physics, 81(1), 109-162 (2009).

[4] F. Bonaccorso, Z. Sun, T. Hasan et al., "Graphene photonics and optoelectronics," Nature Photonics, 4(9), 611$622(2010)$.

[5] C. Cong, T. Yu, K. Sato et al., "Raman Characterization of ABA- and ABC-Stacked Trilayer Graphene," Acs Nano, 5(11), 8760-8768 (2011).

[6] C. H. Lui, Z. Li, K. F. Mak et al., "Observation of an electrically tunable band gap in trilayer graphene,” Nat Phys, 7(12), 944-947 (2011).

[7] E. Delahaye, N. Sandeau, Y. Tao et al., "Synthesis and Second Harmonic Generation Microscopy of Nonlinear Optical Efficient Hybrid Nanoparticles Embedded in Polymer Films. Evidence for Intra- and Internanoparticles Orientational Synergy," The Journal of Physical Chemistry C, 113(21), 9092-9100 (2009).

[8] S. Brasselet, V. Le Floc'h, F. Treussart et al., "In Situ Diagnostics of the Crystalline Nature of Single Organic Nanocrystals by Nonlinear Microscopy," Physical Review Letters, 92(20), 207401 (2004).

[9] M. A. van der Veen, B. F. Sels, D. E. De Vos et al., "Localization of p-Nitroaniline Chains Inside Zeolite ZSM5 with Second-Harmonic Generation Microscopy," Journal of the American Chemical Society, 132(19), 66306631 (2010).

[10] M. A. van der Veen, J. Van Noyen, B. F. Sels et al., "Mapping of the organization of p-nitroaniline in SAPO-5 by second-harmonic generation microscopy," Physical Chemistry Chemical Physics, 12(36), 10688-10692 (2010).

[11] M. A. van der Veen, F. Vermoortele, D. E. De Vos et al., "Point Group Symmetry Determination via Observables Revealed by Polarized Second-Harmonic Generation Microscopy: (1) Theory," Analytical Chemistry, 84(15), 6378-6385 (2012).

[12] M. A. van der Veen, F. Vermoortele, D. E. De Vos et al., "Point Group Symmetry Determination via Observables Revealed by Polarized Second-Harmonic Generation Microscopy: (2) Applications," Analytical Chemistry, 84(15), 6386-6390 (2012).

[13] A. Gasecka, L. Q. Dieu, D. Brühwiler et al., "Probing Molecular Order in Zeolite L Inclusion Compounds Using Two-Photon Fluorescence Polarimetric Microscopy," The Journal of Physical Chemistry B, 114(12), 4192-4198 (2010). 
[14] J. K. Park, S. M. Song, J. H. Mun et al., "Graphene Gate Electrode for MOS Structure-Based Electronic Devices," Nano Letters, 11(12), 5383-5386 (2011).

[15] T. Yoon, W. C. Shin, T. Y. Kim et al., "Direct Measurement of Adhesion Energy of Monolayer Graphene AsGrown on Copper and Its Application to Renewable Transfer Process," Nano Letters, 12(3), 1448-1452 (2012).

[16] A. C. Ferrari, J. C. Meyer, V. Scardaci et al., "Raman spectrum of graphene and graphene layers," Physical Review Letters, 97(18), 187401 (2006).

[17] S. Pisana, M. Lazzeri, C. Casiraghi et al., "Breakdown of the adiabatic Born-Oppenheimer approximation in graphene," Nature Materials, 6(3), 198-201 (2007).

[18] A. Das, S. Pisana, B. Chakraborty et al., "Monitoring dopants by Raman scattering in an electrochemically topgated graphene transistor," Nature Nanotechnology, 3(4), 210-215 (2008).

[19] B. Krauss, T. Lohmann, D. H. Chae et al., "Laser-induced disassembly of a graphene single crystal into a nanocrystalline network," Physical Review B, 79(16), 165428 (2009).

[20] M. A. van der Veen, D. E. De Vos, and T. Verbiest, "Unraveling molecular architecture inside zeolites with second-harmonic generation microscopy," 77580D-77580D (2010).

[21] T. Gokus, R. R. Nair, A. Bonetti et al., "Making Graphene Luminescent by Oxygen Plasma Treatment," Acs Nano, 3(12), 3963-3968 (2009).

[22] G. Eda, Y.-Y. Lin, C. Mattevi et al., "Blue Photoluminescence from Chemically Derived Graphene Oxide," Advanced Materials, 22(4), 505-509 (2010).

[23] Z. Luo, P. M. Vora, E. J. Mele et al., "Photoluminescence and band gap modulation in graphene oxide," Applied Physics Letters, 94(11), 111909 (2009).

[24] H. Zhang, D. Y. Tang, L. M. Zhao et al., "Large energy mode locking of an erbium-doped fiber laser with atomic layer graphene," Opt. Express, 17(20), 17630-17635 (2009).

[25] H. Zhang, D. Tang, R. J. Knize et al., "Graphene mode locked, wavelength-tunable, dissipative soliton fiber laser,” Applied Physics Letters, 96(11), 111112 (2010).

[26] W.-T. Liu, S. W. Wu, P. J. Schuck et al., "Nonlinear broadband photoluminescence of graphene induced by femtosecond laser irradiation," Physical Review B, 82(8), 081408 (2010).

[27] R. J. Stöhr, R. Kolesov, J. Pflaum et al., "Fluorescence of laser-created electron-hole plasma in graphene," Physical Review B, 82(12), 121408 (2010).

[28] J. J. Dean, and H. M. van Driel, "Second harmonic generation from graphene and graphitic films," Applied Physics Letters, 95(26), (2009). 